\title{
Gerencia de la Innovación en Pymes de Nicaragua (Estudio de campo en 26 Pymes de Nicaragua)
}

Omar Antonio Escobar Cerda

Consultor independiente. Residencial Don Bosco, casa E-266, Managua. Correo electrónico: oaec77@yahoo.com, omaraec@yahoo.com

LA INNOVACIÓN, TANTO A NIVEL MACRO COMO A NIVEL MICRO, se vuelve de gran importancia para competir en un mundo globalizado y ayudar a países como Nicaragua a encontrar nuevas vías de crear riqueza que no sean las tradicionales de producir bienes primarios, usar recursos naturales o emplear mano de obra barata. En este artículo se analizan los resultados de una investigación de campo llevada a cabo en Nicaragua en el período de octubre 2007 a febrero 2008. Se entrevistó a 26 Pymes de alto rendimiento de distintos sectores económicos y operando en distintas regiones del país. La mayoría de ellas habían participado en la primera etapa del programa público "Proyecto de apoyo a la innovación tecnológica". El objetivo principal de la investigación fue mostrar cómo estas Pymes manejan y gestionan a nivel interno la innovación. El artículo muestra y analiza los elementos característicos de la gestión de la innovación en este grupo de Pymes. Constituye un enfoque micro de la innovación en un grupo muy especial de Pymes en Nicaragua.

Palabras clave: gerencia-innovación/ gestión-innovación/ Pymes-alto rendimiento

\section{Introducción}

La innovación es el sello distintivo y práctica constante de organizaciones exitosas y extraordinarias. Se le puede encontrar en formas distintas como productos, servicios, procesos, estructuras organizativas y modelos de negocios nuevos o mejorados. La capacidad de cualquier tipo de organización de practicar de forma sistemática y efectiva la innovación es lo que se puede denominar Gerencia de la Innovación.

La Gerencia de la Innovación constituye un conjunto de principios y métodos orientados a promover diferentes tipos de innovación en una organización. Considerando que la capacidad de sobrevivir o crecer de cualquier empresa en el mundo actual está determinada fundamentalmente por dos tipos de estrategias: costos y/o diferenciación, es importante estudiar la experiencia de Pymes nicaragüenses de alto rendimiento con respecto a la promoción de la innovación o de una estrategia de diferenciación. Es esta última estrategia la que provee una ventaja competitiva de más largo plazo. 
El tema de la innovación es relativamente nuevo en Nicaragua. Ha sido en los últimos años promovido a través de un programa público llamado "Proyecto de apoyo a la innovación tecnológica” (PAIT).

Este programa tiene básicamente dos objetivos que se explicarán más adelante: el establecimiento de un sistema nacional de innovación y la promoción de la innovación tecnológica en Pymes locales de alto rendimiento.

Tomando en cuenta la contribución de las Pymes a la economía nacional en términos de PIB y empleo, era interesante desde la formulación del proyecto de investigación conocer de primera mano si PAIT ha mejorado la capacidad para conducir la innovación de un grupo especial de Pymes locales.

La mayor motivación no era solamente ejecutar una evaluación sobre el tema Gerencia de la Innovación en el período en que estas Pymes participaron en PAIT, sino de hecho descubrir el éxito alcanzado por estas empresas en incorporar efectivamente los principios de una gerencia exitosa de la innovación para practicarlos de manera permanente e independiente.

Por independiente nos referimos a la habilidad de estas Pymes de desarrollar proyectos de innovación sobre su propio conocimiento adquirido, y no necesariamente trabajando aisladamente, sino activamente buscando alianzas con otras empresas u organizaciones. El tema de las alianzas es un indicador clave de su compromiso y búsqueda de formas más sofisticadas de innovación que trasciendan la simple adopción de tecnología extranjera.

Se pensó que era de gran importancia examinar los tipos de innovación más practicados por estas Pymes de alto rendimiento, considerando el énfasis puesto por PAIT en la innovación tecnológica. Teniendo en cuenta que algunas de estas Pymes pueden ser líderes o destacan en sus respectivos mercados y sectores, es de gran valor describir cómo pueden estar marcando la pauta para las prácticas de innovación en el ambiente local.

Este estudio es pionero al arrojar luz sobre un asunto de capital importancia tanto para el gobierno como para el sector privado local. Las fuerzas de la globalización y el proceso de integración con el resto de Centroamérica hacen que la transformación de prácticas de Pymes locales sea una necesidad impostergable para Nicaragua. Es de especial importancia ayudar a las Pymes más avanzadas a entender y adoptar principios de innovación que las empujen hacia mayores niveles de competitividad.

\section{Objetivos de investigación e hipótesis}

El objetivo de este artículo, basado en una investigación de campo, es permitir comprender el estado de Gerencia de la Innovación en Pymes de alto rendimiento en Nicaragua. Es un estudio cualitativo sobre cómo un grupo especial de Pymes en Nicaragua está manejando distintos aspectos que conforman una exitosa Gerencia de la Innovación. La investigación se basa en una amplia descripción de los aspectos centrales que determinan la Gerencia de la Innovación por medio del análisis de una muestra seleccionada de Pymes de alto rendimiento en Nicaragua. 
Las preguntas subyacentes en el estudio y las correspondientes hipótesis para cada pregunta son:

1. ¿Promueven las Pymes de alto rendimiento en Nicaragua distintos tipos de innovación a nivel interno?

H1: La práctica de innovación se limita a la adopción de tecnología e innovación de producto.

2. ¿Se practica la innovación radical en estas Pymes de alto rendimiento?

H2: La innovación radical es raramente practicada contrario a la innovación gradual.

3. ¿Sostiene este grupo especial de Pymes alianzas de cualquier índole para desarrollar proyectos de innovación?

H3: Las Pymes de alto rendimiento en Nicaragua sostienen pocas alianzas relacionadas a proyectos de innovación.

4. ¿Qué tan exitosas son estas Pymes de alto rendimiento al desarrollar sus proyectos de innovación?

H4: Las Pymes de alto rendimiento en Nicaragua muestran una pobre práctica de Gerencia de la Innovación.

\section{Metodología}

Método de investigación: Considerando la negativa tradicional de empresas de suministrar información cuantitativa sobre sus operaciones, se pensó que la mejor manera de enfocar el tema de Gerencia de la Innovación sería a través del uso de información cualitativa. Esto hizo menos complicado el persuadir a las Pymes seleccionadas para suministrar información.

Cuestionario: El cuestionario diseñado y usado para recoger la información relevante consistía de 17 preguntas divididas en cinco secciones: Información General de la empresa, Estrategia de Innovación, Cultura y Organización de la Innovación, Procesos del ciclo de vida de la innovación y Resultados de la innovación. La base del cuestionario fue un cuestionario de 40 preguntas utilizado para evaluar la innovación que es aplicado y promovido por la Unión Europea bajo la iniciativa "Europe Innova”. Se redujo significativamente para hacer las entrevistas más rápidas y comprensibles.

Selección de la muestra: Las empresas seleccionadas tenían que cumplir con dos requisitos básicos para ser parte del estudio. Primero, tenían que estar dentro de la definición de Pymes de acuerdo a las categorías definidas por el MIFIC. Segundo, estas Pymes tenían que ser consideradas sobresalientes o de alto rendimiento en el país. Para cumplir este segundo requisito, 24 Pymes fueron seleccionadas de los beneficiarios del programa público PAIT. Para participar en este programa público, las Pymes tenían que cumplir con una serie de criterios de rendimiento financieros y económicos que aseguraban que eran empresas por encima del promedio en sus respectivos sectores. 
Las otras dos empresas entrevistadas fueron elegidas a través de un contacto personal y de un programa público del INDE (Instituto Nicaragüense de Desarrollo) dirigido a mejorar capacidades tecnológicas en Pymes locales. En total se entrevistó a 26 Pymes que representan a una gran variedad de sectores o industrias locales. Se pretendía disponer de una muestra variada en cuanto a los sectores de operación de las empresas.

Los sectores representados, en orden de importancia, son: textiles, madera y muebles, productos metálicos, agroindustria, panadería, producción de fármacos, pesca, lácteos, cuero y zapatos, tecnología de información, producción de sal, productos plásticos, laboratorio para alimentos, vidrio y productos de madera, agroindustria/metales y materiales de construcción.

De las 26 Pymes, 15 eran empresas medianas, 10 pequeñas y una micro. En cuanto a la localización, 18 empresas eran de Managua y ocho de otras ciudades de Nicaragua. Se buscó entrevistar a mayor cantidad de empresas de otras regiones del país pero por problemas de comunicación telefónica y de disponibilidad no fue posible.

Recolección de datos: La investigación comprendió el período del 6 de noviembre de 2007 al 2 de marzo de 2008. Del programa PAIT se recibió una lista de 39 empresas participantes en la primera fase del proyecto. Posteriormente se procedió a contactar a las empresas de la lista. Se entrevistó a 26 Pymes; 15 de ellas fueron entrevistadas por teléfono y 11 fueron entrevistadas in situ. En promedio, las entrevistas telefónicas duraron 10 minutos y las visitas 30 minutos. La mayoría de los entrevistados fueron los gerentes o dueños de estas empresas y en los dos únicos casos en que no fue así, se entrevistó a personas con importantes responsabilidades administrativas y con suficiente conocimiento para responder a las preguntas hechas.

Análisis estadístico: La información recogida se analizó con la ayuda del programa estadístico SPSS versión 12. A fin de analizar la información se recurrió a estadísticas descriptivas, representando los resultados por medio de frecuencias, gráficos, tablas y pasteles.

\section{Antecedentes}

Existe una evidente falta de estudios e investigaciones sobre el tema de la innovación, en general sobre la economía de Nicaragua y específicamente relacionados con las Pymes del país. Toda la información referente al tema se puede obtener de algunos sitios web gubernamentales porque el conocimiento y comprensión adquirida se ha realizado en los últimos años a través de programas públicos como PAIT, que constituye el primer esfuerzo serio del gobierno por promover la innovación en Nicaragua. En la comunidad académica el énfasis es puesto abrumadoramente en temas como "Pymes y competitividad" y sólo algunas referencias muy someras se hacen con respecto al tema de la innovación.

La idea de apoyar el desarrollo del sector de Pymes en Nicaragua surgió en el MIFIC como parte de la visión del presidente Enrique Bolaños (2002-2007) para aumentar la competitividad de este importante sector. El BID concedió un préstamo de US\$2.9 millones 
de dólares al gobierno nicaragüense (Proyecto de apoyo a la innovación tecnológica, 2004). El préstamo se usó para lanzar el "Proyecto de apoyo a la innovación tecnológica" conocido como PAIT, que se concibió como un proyecto piloto para mejorar la competitividad de las Pymes locales.

El objetivo de PAIT fue "establecer las bases de un sistema nacional de innovación, el cual también promueva la innovación tecnológica en las Pymes locales”. Éste, a través del mecanismo de liberación de fondos compartidos, concedía financiamiento no reembolsable para proyectos de innovación tecnológica en Pymes locales y para proyectos de mejora de oferta tecnológica tanto en laboratorios independientes como en laboratorios de universidades.

Es importante aclarar que los fondos de PAIT fueron concedidos ante la presentación de propuestas concretas de proyectos, y el financiamiento complementario fue aportado por el beneficiario. Habían dos modalidades: las empresas individuales recibían de PAIT el 60\% y aportaban el restante 40\%; las empresas asociadas recibían de PAIT el 80\% y aportaban el restante 20\%. De esta forma, las Pymes beneficiarias en la primera fase de PAIT han contribuido con más de un millón de dólares americanos, con lo que la inversión total hecha asciende a cerca de cinco millones de dólares americanos.

PAIT proveyó fondos fundamentalmente para que las Pymes locales mejoraran su tecnología productiva, pero también dirigió fondos para innovación organizativa, acceso a nuevos mercados, mejora de tecnología de información, sistemas contables computarizados, reingeniería, registro de patentes y marcas, empacado, código de barra, control de calidad y contactos con potenciales clientes.

\section{Marco teórico}

Para desarrollar un efectivo proceso de innovación, los gerentes de Pymes deben preocuparse no solamente por productos, tecnología y procesos, sino también por la cultura de la organización, sus normas, valores y creencias (Gunasekaran, 1996). Hay una fuerte necesidad de crear un clima que induzca a la creatividad (Pervaiz, 1998) con un claro énfasis externo en múltiples participantes.

Los autores identifican cuatro tipos de innovación citadas en la literatura: innovación de productos, innovación de procesos, innovación organizativa o de recursos humanos y la de modelos de negocios (Damanpour, 1996). Éstos se pueden reagrupar en dos grandes grupos: 1) productos, sistemas tecnológicos y procesos y 2) diseño de trabajo, organización de trabajo, recursos humanos y modelos de negocios.

Estas dos categorías pueden ser innovaciones radicales o discontinuas, y graduales o continuas. Los distintos tipos de innovación se explican detalladamente a continuación:

Innovación radical o discontinua: Los mercados aparecen como de la nada, repentinamente se crean nuevas fuentes de riqueza. Tiene sus raíces en discontinuidades tecnológicas como la que permitió a Motorola ganar fama con la primera generación de teléfonos celulares. Sin 
embargo, no se limita a productos. Se puede definir como cualquier nuevo y desconocido producto, tecnología, proceso, estructura organizativa y modelo de negocio.

Innovación gradual o continua: Es más el tipo continuo y sistemático de innovación. Busca lograr una ligera diferenciación en productos existentes así como procesos, tecnología, estructura organizativa y modelos de negocios ya probados.

Innovación de producto: Es la creación de un nuevo producto o el mejoramiento de uno existente. El objetivo puede ser mejora de rendimiento, reducción de costos y ampliación de usos.

Innovación de procesos: Hace los procesos más eficientes tanto en mercados nuevos como establecidos.

Innovación de modelos de negocios: Reformula una propuesta de valor establecida al cliente o el rol establecido de una empresa en la cadena de valor o ambos.

La extensión de esta lista puede ser problemática. ¿Cómo deciden gerentes y ejecutivos dónde concentrarse? En un tiempo la noción de competencias básicas se invocó para solucionar el problema: escoger los puntos fuertes para enfocar los recursos de manera coherente. No obstante, las empresas han descubierto que ser el mejor en algo no garantiza una ventaja competitiva. Dichas ventajas son valiosas sólo si cambian las preferencias del consumidor.

El objetivo central de la innovación es ayudar a mejorar el rendimiento de corto plazo y mantener el de largo plazo de cualquier organización. Cualquier tipo de innovación por sí mismo no puede completamente o continuamente preservar el deseado nivel de rendimiento en el curso del tiempo. La concentración en un solo tipo de innovación puede generar un impacto muy limitado.

La consistencia de la actividad innovadora tendrá con el curso del tiempo un impacto positivo en el rendimiento porque los tipos de innovación son interdependientes y la introducción de cualquiera de los tipos mejora el valor de los otros (Roberts \& Amit, 2003). Por lo tanto, una muy buena estrategia sería la promoción de un amplio abanico de tipos de innovación. Las organizaciones deberían buscar innovar en todas las áreas y diferenciarse de la norma.

\section{Resultados y discusión}

\subsection{Estrategia de innovación}

Hipótesis 1: "La práctica de innovación se limita a la adopción de tecnología e innovación de producto". 
Cuadro 1. Estrategia de innovación - características

\begin{tabular}{|c|c|c|}
\hline Estrategia de innovación & $\begin{array}{c}\text { Frecuencia } \\
\text { (Número de Pymes) }\end{array}$ & Porcentaje \\
\hline Innovación de producto o proceso & 19 & 73.1 \\
Innovación de producto/proceso/organizativa & 6 & 23.1 \\
Innovación de producto/proceso/modelo de negocio & 1 & 3.8 \\
Total & 26 & 100.0 \\
\hline
\end{tabular}

Fuente: Elaboración propia

Todas las empresas entrevistadas expresaron que realizaban esfuerzos para practicar algún tipo de innovación. Una clara mayoría de empresas promovía únicamente la innovación circunscrita al ámbito de creación o mejora de productos. El área de innovación de procesos o producción también recibió mucha atención tanto de parte del PAIT como de las Pymes. La innovación organizativa o de modelos de negocios fue escasa y realmente descuidada por las Pymes entrevistadas.

No se tiene mucha información acerca de los criterios usados por estas Pymes para decidir qué tipo de innovación se promovía. Todas estas Pymes practican la innovación de producto y/o proceso. Un alto porcentaje $(73.1 \%)$ ha enfocado sus esfuerzos únicamente en las áreas de producto y proceso. Una minoría de estas Pymes acompaña sus esfuerzos de innovación en producto/producción con proyectos de innovación organizativa y/o modelos de negocios. Éstas son más la excepción que la regla.

El predominio de las innovaciones de tipo técnico como producto y/o proceso confirma la hipótesis 1. Todas estas Pymes la practican y sólo unas cuantas han identificado oportunidades e incentivado las innovaciones de tipo administrativo como las organizativas y modelos de negocios. De acuerdo a este patrón de prácticas de innovación, se puede afirmar que estas Pymes no entienden la necesidad y gran potencial de combinar distintos tipos de innovación durante las distintas fases de desarrollo de un mercado.

Hipótesis 2: "La innovación radical es raramente practicada contrario a la innovación gradual".

Cuadro 2. Existencia de proyectos de innovación radical en últimos cuatro años

\begin{tabular}{|c|c|c|}
\hline $\begin{array}{c}\text { Existencia de proyectos de innovación } \\
\text { radical en últimos cuatro años }\end{array}$ & $\begin{array}{c}\text { Frecuencia } \\
\text { (Número de Pymes) }\end{array}$ & Porcentaje \\
\hline Sí & 10 & 38.5 \\
No & 16 & 61.5 \\
Total & 26 & 100.0 \\
\hline
\end{tabular}

Fuente: Elaboración propia 
Más de la mitad de estas Pymes no desarrolló ningún proyecto de innovación radical en los últimos cuatro años. Sin embargo, existe una significativa cantidad de estas empresas (casi 40\%) que sí ha desarrollado proyectos de innovación radical. De acuerdo a la información recogida, algunas de estas Pymes de alto rendimiento estaban creando y lanzando al mercado productos y/o servicios enteramente nuevos o creando prácticas laborales y estructuras organizativas verdaderamente únicas en el país.

La mayoría de proyectos de innovación radical cayeron en la categoría de producto (80\%) y muy pocos en las áreas de modelos de negocios e innovaciones organizativas (20\%). Elénfasis de los proyectos de innovación radical en el área de producto es nuevamente resultado de la práctica general de innovación, que dirige y concentra casi todos sus recursos en este tipo de innovación particularmente.

De las Pymes que habían desarrollado proyectos de innovación radical en los últimos cuatro años, la mayoría tenía uno o dos proyectos (80\%). El resto (20\%) había ejecutado tres proyectos de innovación radical. Muy pocos proyectos de esta naturaleza fallaron en entregar los resultados esperados, alcanzando gran efectividad en su ejecución. De hecho, solamente dos de las Pymes con este tipo de proyectos de innovación fracasaron. Uno de estos casos fue el de una empresa que creó un nuevo licor en base a semilla de jícaro, pero fracasó en la comercialización porque no encontraron puntos de distribución como supermercados, etc.

En cuanto a la innovación gradual, todas las Pymes entrevistadas manifestaron practicarla de alguna forma. Esta no fue ninguna sorpresa, puesto que se esperaba de antemano que estas Pymes de alto rendimiento practicaran algún tipo de innovación por lo menos en su forma más elemental y común como la innovación gradual o continua.

En este tipo de proyectos de innovación nuevamente se manifiestan las mismas características de la estrategia de innovación practicada. La mayor parte de los proyectos de innovación graduales estaban relacionados con el área de productos/procesos o con alguna combinación de éstos. Los mismos patrones en la práctica, tanto de proyectos de innovación radicales como graduales, indica que para la mayoría de estas Pymes es como una primera etapa natural promover la innovación relacionada al ámbito de creación o mejora de productos/ procesos.

Se debe entender que el área de producto/proceso es la más visible y donde las empresas de distintos sectores industriales ponen mayor énfasis y empeño para alcanzar una ventaja competitiva. Por lo tanto, en países donde los esfuerzos de promoción de la innovación apenas comienzan, es lógico y comprensible que cuando las empresas locales, especialmente Pymes e incluso las de mejor rendimiento, empiezan a familiarizarse con la innovación, automáticamente orienten sus esfuerzos hacia el área de productos/procesos.

La mayoría de estas Pymes tenía más de un proyecto de innovación gradual desarrollado en los últimos cuatro años (84.6\%). Es claramente menos complicado desarrollar proyectos de innovación gradual en comparación con los de innovación radical. Los proyectos de innovación gradual demostraron ser altamente efectivos para mejorar la ventaja competitiva 
de muchas de estas Pymes. De hecho, no hubo un solo fracaso entre estos proyectos. Todos fueron desarrollados exitosamente.

Lamentablemente, no se pudo conseguir información acerca de la forma en que las Pymes que desarrollaban los dos tipos de innovación combinaban ambos tipos para hacerle frente a las exigencias de las distintas etapas del desarrollo de un mercado. En general, sólo se puede afirmar que estas Pymes fueron exitosas - con algunas excepciones en el caso de la innovación radical- en promover la innovación en ciertas áreas y obtener resultados satisfactorios.

Considerando la evidencia analizada se puede negar la hipótesis 2, puesto que la existencia de 10 Pymes de alto rendimiento en un grupo de 26 entrevistadas (38,5\%) desarrollando proyectos de innovación radical constituye un porcentaje significativo para concluir que la innovación radical no es rara en comparación con la gradual.

\subsection{Organización y cultura de innovación}

Hipótesis 3: "Las Pymes de alto rendimiento en Nicaragua sostienen pocas alianzas relacionadas a proyectos de innovación”.

Cuadro 3. Existencia de alianzas

\begin{tabular}{|c|c|c|}
\hline Existencia de alianzas & Frecuencia (Número de Pymes) & Porcentaje \\
\hline Sí & 11 & 42.3 \\
No & 15 & 57.7 \\
Total & 26 & 100.0 \\
\hline
\end{tabular}

Fuente: Elaboración propia

La mayoría de estas Pymes no tenía ningún tipo de alianza. Las Pymes entrevistadas que sostenían alianzas estaban en su mayor parte involucradas en alianzas temporales e informales con empresas complementarias. El propósito de estas alianzas, en el caso de nueve de estas Pymes, era fundamentalmente la administración conjunta de cadenas de suministro, acuerdos de distribución o comercialización, manufactura conjunta y subcontratación de la producción.

Solamente dos empresas, de las once que sostenían alianzas, estaban involucradas en alianzas de transferencia de conocimientos con ONG locales y acuerdos de colaboración con universidades.

La mayoría de estas Pymes con algún tipo de alianza en los pasados cuatro años sólo tenían un socio regular $(63.6 \%)$ y un pequeño grupo tenían más de un socio regular (36.4\%). Al analizar los datos recogidos, se puede afirmar que la hipótesis 3 es verdadera. Menos de 
la mitad de las Pymes entrevistadas sostenían alianzas y de las que sostenían alianzas, la mayoría solamente tenían alianzas con un socio regular. Además, la mayoría de las Pymes con alianzas estaban comprometidas en acuerdos de tipo operativo, los que tienen potencial mayormente para la innovación gradual. En este grupo, sólo dos Pymes colaboraban con una ONG local y una universidad, las que constituyen relaciones con potencial para la innovación radical.

\subsection{Procesos del ciclo de vida de la innovación}

Hipótesis 4: "Las Pymes de alto rendimiento en Nicaragua muestran una pobre práctica de Gerencia de la Innovación”.

La primera variable que se evaluó en este acápite fue la de tiempo-mercado. Esta variable mide el tiempo que transcurre desde la concepción de una idea para un producto y/o servicio hasta su introducción en el mercado. Se les preguntó a todas las Pymes de alto rendimiento si sabían el valor tiempo-mercado para algunos o todos los productos y/o servicios innovados en los últimos años. Sólo una empresa manifestó no poseer conocimiento acerca de esta variable. El resto sí sabía cuánto tiempo había llevado concebir y lanzar productos y/o servicios innovados al mercado.

El conocimiento de estos valores de parte de casi todas las Pymes puede indicar una buena gestión del proceso innovador, ya que la atención a restricciones de tiempo es un deber para desarrollar exitosamente la innovación. Altos costos, productos y servicios obsoletos así como pérdida de oportunidades, pueden surgir de un pobre conocimiento de esta variable y de la falta de atención al respecto.

Un conocimiento aproximado o exacto de la variable tiempo-mercado, basado en experiencias pasadas, también les permite conducirse mejor y anticipar los retrasos típicos del proceso de innovación así como poder coordinar más eficientemente las diferentes actividades emprendidas por distintas unidades dentro de la misma empresa.

Cuadro 4. Ideas, proyectos y toma de decisiones

\begin{tabular}{|c|c|c|}
\hline Tipo de estructura organizativa & $\begin{array}{c}\text { Frecuencia (Número } \\
\text { de Pymes) }\end{array}$ & Porcentaje \\
\hline Equipo multidisciplinario & 12 & 46.2 \\
Negocio familiar & 11 & 42.3 \\
Centrado en dueño y/o gerente general & 3 & 11.5 \\
Total & 26 & 100.0 \\
\hline
\end{tabular}

Fuente: Elaboración propia

Era importante descubrir cómo eran las estructuras predominantes en cada una de estas Pymes para tomar decisiones con respecto a nuevas ideas, proyectos, etc. Comparadas 
individualmente cada una de estas categorías, se puede observar que los "equipos multidisciplinarios" son una ligera mayoría.

Probablemente, el hecho de que la mayoría de Pymes entrevistadas eran empresas medianas explica la existencia de este tipo de estructura, ya que cuanto más grande la empresa, más descentralizada y diversificada su estructura. Es en este tipo de estructura que se tiene capacidades más desarrolladas para empezar, alimentar y desarrollar exitosamente el proceso de innovación porque las decisiones son tomadas por un grupo de profesionales y técnicos cualificados y no solamente por la familia propietaria o el dueño y/o gerente como es el caso en las más pequeñas.

La naturaleza de la interacción de los empleados de distintos niveles entre si mismos y con la gerencia, en estas empresas no se conoce a profundidad. También se debe mencionar que una gran parte de estas Pymes todavía son propiedad de familias. En muchas de ellas el gerente es el fundador o miembro del núcleo familiar propietario. Sin embargo, ha habido un buen número de ellas que han emprendido reformas a lo interno, lo cual ha creado estructuras más abiertas al punto de tener un número significativo de ellas con "equipos multidisciplinarios". Estos equipos o estructuras funcionan tanto para actividades operativas como para proyectos de innovación. A pesar de lo mencionado, este es un terreno en el que aún se debe mejorar mucho para poder impulsar más eficientemente la innovación.

Cuadro 5. Existencia de incentivos para la innovación de los empleados

\begin{tabular}{|c|c|c|}
\hline $\begin{array}{c}\text { Existencia de } \\
\text { incentivos }\end{array}$ & $\begin{array}{c}\text { Frecuencia (Número de } \\
\text { Pymes) }\end{array}$ & Porcentaje \\
\hline Sí & 18 & 69.2 \\
No & 8 & 30.8 \\
Total & 26 & 100.0 \\
\hline
\end{tabular}

Fuente: Elaboración propia

Viendo el cuadro 5 se puede afirmar que una gran parte de las Pymes de alto rendimiento están concientes del gran significado que encierra el ofrecer estímulos o recompensas para despertar la creatividad, la toma de riesgos y el compromiso hacia los proyectos de innovación por parte de los empleados. Este es un elemento clave de la administración de recursos humanos, lo que representa otro claro indicador de una buena gestión de la innovación.

La mayoría de estas Pymes usan "dinero o bonos" o estímulos financieros como estrategia para comprometer a sus empleados. Algunas Pymes expresaron pagar mejores salarios comparados a la competencia para estimular este tipo de actitudes. Otro grupo ofrece oportunidades de inserción social o capacitaciones para alcanzar el mismo objetivo.

El perfeccionamiento de los sistemas de incentivos por parte de la gerencia de estas Pymes debería ser un objetivo a perseguir. El hecho de que hay empresas en este grupo que 
consideran suficiente el pagar mejores salarios, ofrecer capacitaciones o dar opciones de inserción social a sus empleados, indica que todavía no se ha madurado en el sentido de entender que para desarrollar una verdadera cultura de búsqueda de riesgos y aventuras, se debe construir un sistema de incentivos poderosos que permita compartir las ganancias de los proyectos de innovación con los empleados que más han contribuido a ello.

Cuadro 6. Existencia de investigación y desarrollo

\begin{tabular}{|c|c|c|}
\hline Existencia de I+D & $\begin{array}{c}\text { Frecuencia (Número } \\
\text { de Pymes) }\end{array}$ & Porcentaje \\
\hline Sí & 8 & 30.8 \\
No & 18 & 69.2 \\
Total & 26 & 100.0 \\
\hline
\end{tabular}

Fuente: Elaboración propia

50 Una amplia mayoría de estas Pymes no realiza ningún tipo de actividad de I+D. En general, las Pymes que respondieron afirmativamente no poseían formalmente un departamento de I+D. De las Pymes con estas actividades, un alto porcentaje las llevaba a cabo a través del gerente general y/o algún otro empleado cercano al gerente.

La existencia de esta actividad -no necesariamente limitada a la operación formal de un departamento- es crucial como una fuente de nuevas ideas y conocimiento que aportan en gran medida a la gestión de la innovación. Es de capital importancia que una empresa conduzca sus propias actividades de I+D, aunque sea de manera informal, para no depender de agentes externos en la generación y desarrollo de conocimiento.

La mayoría de los equipos de I+D operando en estas Pymes estaban conformados por no más de tres personas. En estos casos, era fundamentalmente la responsabilidad del gerente junto con algún socio o empleado de confianza investigar sobre distintos temas, recoger información y proponer nuevas líneas de productos, servicios o soluciones a distintos problemas existentes en la empresa. Esta era típicamente la situación en las empresas más pequeñas, contrario a la situación de las más grandes, en las que era más probable encontrar, dependiendo de su sector de operación, un departamento formal de I+D.

En cuanto al aspecto de financiamiento para proyectos de innovación, como se explicó anteriormente, todas las Pymes beneficiarias de PAIT, utilizaron una mezcla en distintas medidas de recursos propios con recursos otorgados como no reembolsables por el gobierno. Esto fue durante su participación en el programa, después sólo contaban con sus propios recursos para desarrollar nuevos proyectos de innovación. En cuanto a las dos Pymes que no participaron en PAIT, todos sus esfuerzos de innovación fueron financiados enteramente por ellos.

Sin lugar a dudas, esta es la situación de la mayor parte de Pymes en el país. No hay financiamiento bancario o de otras fuentes disponible para estos fines. 
Cuadro 7. Lanzamiento exitoso de productos innovados al mercado en últimos cuatro años

\begin{tabular}{|c|c|c|}
\hline $\begin{array}{c}\text { Lanzamiento exitoso de productos } \\
\text { innovados en últimos } \mathbf{4} \text { años }\end{array}$ & $\begin{array}{c}\text { Frecuencia (Número de } \\
\text { Pymes) }\end{array}$ & Porcentaje \\
\hline Sí & 25 & 96.2 \\
No & 1 & 3.8 \\
Total & 26 & 100.0 \\
\hline
\end{tabular}

Fuente: Elaboración propia

Se puede observar que es casi perfecto en estas Pymes el lanzamiento de productos innovados, ya sea nuevos o mejorados, en los últimos cuatro años. Como ya se había mencionado anteriormente, el único caso fallido fue el del licor en base a semilla de Jícaro de "Industrias Reichel". El producto fue incluso patentado. El problema enfrentado fue el enfoque de comercialización. Trataron de vender ellos mismos el producto en puntos como supermercados pero no encontraron aceptación. Una mejor estrategia hubiera sido conceder el producto en licencia a una empresa local reconocida en el sector, que al hacer uso de sus relaciones establecidas con distribuidores y clientes, podría haber tenido éxito en vender el producto.

Relacionado con este aspecto del mercadeo de productos innovados, se debe mencionar que la mayor parte de estos productos se vendían en el mercado nacional. Muy pocos de ellos se lograban vender en mercados regionales como Centroamérica y en los internacionales eran también muy bajos sus niveles de venta. El asunto de la internacionalización y las exportaciones de las Pymes nicaragüenses es algo que sigue pendiente y con rezagos con respecto a otros países cercanos. Esto es válido tanto para sus productos funcionales como para los innovados. Este mismo problema puede ofrecer la explicación para tanto éxito en los proyectos de innovación y lanzamiento de productos innovados, porque el mercado local es el menos exigente y sofisticado.

Cuadro 8. Creación de marcas en últimos cuatro años

\begin{tabular}{|c|c|c|}
\hline $\begin{array}{c}\text { Creación de marcas por empresa } \\
\text { en últimos cuatro años }\end{array}$ & $\begin{array}{c}\text { Frecuencia (Número } \\
\text { de Pymes) }\end{array}$ & Porcentaje \\
\hline Sí & 15 & 57.7 \\
No & 11 & 42.3 \\
Total & 26 & 100.0 \\
\hline
\end{tabular}

Fuente: Elaboración propia

La creación de marcas como una señal de identidad para productos nuevos fue practicada por una ligera mayoría de las Pymes entrevistadas. Del grupo de Pymes de alto rendimiento que crearon marcas, muchas usaban el nombre de la empresa como marca de ciertas líneas de productos. La mayoría solamente crearon una marca y en gran parte con éxito en los últimos cuatro años. Este bajo ritmo de creación de marcas puede ser consecuencia de servir sólo al mercado nacional. 
Después de presentar todas estas variables se puede revisar la hipótesis 4 nuevamente. En aspectos como tiempo-mercado, mercadeo de productos innovados, creación y manejo de marcas, sistemas de incentivos y tipo de estructuras de decisión, ha salido bastante bien este grupo especial de Pymes. Es apropiado decir que dentro de sus contextos específicos, sus prácticas de gestión de la innovación han demostrado ser exitosas. Por tal razón, la hipótesis 4 es falsa. Sin embargo, esto no quiere decir que estas Pymes no necesiten perfeccionar sus prácticas. En realidad deben mejorar mucho, la mayoría de ellas ni siquiera es conciente de la existencia de los distintos componentes de un eficiente sistema de innovación. Solamente se observó que sus prácticas no son pobres como se afirmó al iniciar la investigación.

\section{Conclusiones}

Esta investigación fue pionera en explorar a profundidad un tema profundidad del que se necesitan sacar muchas lecciones que aporten a aumentar el interés, conocimiento y prácticas de innovación en Nicaragua. Es importante aclarar al respecto que los resultados de esta investigación no se deben generalizar porque están basados en una muestra muy pequeña, selecta y variada de Pymes. Las Pymes que fueron entrevistadas durante esta investigación operan en una gran variedad de sectores, la mayoría de ellos industriales, pero también en sectores de servicio, lo que permite analizar la práctica de la innovación, en entornos muy diversos.

Se repasará brevemente los principales resultados de la investigación. Primero, La práctica de la innovación en este grupo especial de Pymes se limita casi totalmente a la creación de nuevos productos o a la mejora de productos existentes así como a la mejora del proceso de producción, es decir, a las innovaciones de tipo técnico. Muy pocas han identificado el gran potencial de las innovaciones de tipo administrativo u organizativo como prácticas laborales y modelos de negocios. Probablemente sea el resultado de pocos casos de éxito conocidos en estas áreas y de las áreas prioritarias establecidas por PAIT.

Segundo, se descubrió que había un grupo importante de estas Pymes que sí desarrollaba proyectos de innovación radical (38,5\%). Más sorprendente aún fue descubrir el altísimo nivel de éxito obtenido en el desarrollo de este tipo de innovación. Hay algunas razones que pueden explicar estos resultados. Los datos sobre innovación radical pudieron haber sido inflados por falta de comprensión sobre la diferencia entre este tipo de innovación y la innovación gradual. Es probable que muchos de los entrevistados se hayan referido realmente a la innovación gradual cuando respondieron sobre este tipo de innovación.

Se sospecha eso porque previo a las entrevistas no se les explicó los conceptos generales a ser usados, sólo se les mencionó el propósito de la misma. Además, el corto tiempo del que se disponía para entrevistar no hacía posible profundizar en detalles y explicaciones, lo que pudo conducir a malos entendidos.

Por otro lado, se piensa que hubo factores circunstanciales que también explican los extraordinarios resultados de proyectos de innovación radical. Todas estas Pymes contaron con la asistencia de consultores provistos por PAIT, los que aportaron conocimientos y experiencia para desarrollar los distintos proyectos de innovación. Esta ayuda especial con 
la que contaron en determinado período puede explicar el alto nivel de éxito. Por último, la mayoría de los productos innovados se introducían al mercado nacional y unos cuantos en mercados regionales e internacionales. El mercado nacional es menos sofisticado en cuanto a las preferencias de los consumidores y tiene menor oferta de productos en muchos nichos, lo que pudo contribuir a tan alto nivel de éxito en los proyectos de innovación radical.

Tercero, se descubrió que menos de la mitad de las Pymes entrevistadas (42,3\%) estaban comprometidas en algún tipo de alianza. De este grupo, la mayoría (63,6\%) sólo tenía un tipo de alianza. El resto tenía alianzas múltiples. La mayor parte de las alianzas era de naturaleza comercial o para fines operativos, y muy pocas Pymes tenían relaciones de colaboración con universidades y ONG locales.

Finalmente, se puede afirmar que este grupo de Pymes de alto rendimiento tiene buenas prácticas de gestión de la innovación. Variables como la existencia de sistemas de incentivos, tiempo-mercado, creación y gerencia de marcas, y mercadeo de productos innovados muestran que han tenido mucho éxito en los últimos años. Nuevamente se debe recordar que hay factores circunstanciales y de estructura de mercado que pueden explicar el éxito conseguido. Por tal razón, se debe pensar en darle seguimiento a este estudio para observar la evolución de estas Pymes y ayudarlas a alcanzar conciencia sobre los componentes de un eficiente sistema de gestión de la innovación para que con el tiempo mejoren su rendimiento en áreas deficientes en la actualidad.

\section{Recomendaciones}

En cuanto a la conducción de nuevas investigaciones sobre gestión de la innovación en Nicaragua, se deben considerar las siguientes recomendaciones:

- Tratar de coordinar el levantamiento de la información con respaldo total de las instituciones correspondientes para presionar a las Pymes seleccionadas a suministrar mayor información.

- Extender el tiempo de las entrevistas para poder explicar previamente conceptos claves para la comprensión de las preguntas y obtener la mayor cantidad de detalles posibles.

- Entrevistar no sólo a los gerentes, sino también a gerentes medios o funcionales y técnicos que puedan aportar otras visiones e información con el fin de enriquecer el estudio.

En cuanto a la construcción de un mejor entorno para la difusión de la innovación a través de políticas públicas más eficientes, se hacen las siguientes propuestas:

- Se deben reformar los mercados de capitales para aumentar el acceso a fondos de Pymes que no están siendo atendidas por el sector bancario. Para esto es importante también el diseño de políticas fiscales que busquen incrementar la liquidez de las Pymes, sobre todo en sus primeros años de existencia para que puedan disponer de mayores recursos y emprender proyectos de innovación. Se debe pensar en atraer y crear una red de 
inversores ángeles, locales o extranjeros, que faciliten los fondos que tanto necesitan las Pymes para innovar y crecer.

- Se debe fomentar la cultura de colaboración y las capacidades de asociación de las Pymes locales. Primero, se debe buscar acercar la Universidad a la empresa y viceversa. Esto se puede hacer estableciendo parques científicos y tecnológicos. Segundo, la creación de incubadoras de empresas es crucial para crear una cultura de colaboración desde el nacimiento de las empresas y despertar la actitud emprendedora que es un ingrediente indispensable para la búsqueda de innovación. Tercero, la formación de clústeres industriales o de servicios puede abonar al desarrollo de las capacidades asociativas de las empresas.

- Se debe comenzar una agresiva campaña de internacionalización de las Pymes. Para lograr esto se debe invertir fuertemente en infraestructura como aeropuertos, puertos y carreteras. Por el lado de las Pymes, se debe buscar aprovechar las oportunidades que ofrecen los tratados de libre comercio que ha firmado Nicaragua en los últimos años.

\section{Agradecimientos}

Hay muchas personas y organizaciones a las que debo agradecer por la realización del proyecto de investigación que es la base del presente artículo. Primero, agradezco a la Lic. Ana María Angulo por la información suministrada, la que sirvió para contactar a parte de las empresas participantes en la primera fase de PAIT. Segundo, mis sinceros agradecimientos a todas las empresas, que de buena voluntad aceptaron brindar algo de su valioso tiempo para contestar el cuestionario utilizado. Finalmente y no menos importante, agradezco al DAAD (Servicio alemán de intercambio académico), el cual por medio de la beca otorgada hizo posible que yo me embarcara en tan importante etapa de mi vida como la realización de estudios en Alemania que condujeron posteriormente a la investigación de campo.

\section{Referencias bibliográficas}

DAMANPOUR, F. (1996). "Organizational Complexity and Innovation: Developing and Testing Multiple Contingency Models”. En Management Science. 42:693-716, Philadelphia.

GUNASEKARAN, A. (1996). "A Conceptual Framework for Managing Congestion in Manufacturing”. En International Journal of Production Economics. Vol. 45, Edición 1-3:111-119, California.

PERVAIZ, A. (1998). "Culture and Climate for Innovation”. En European Journal of Innovation Management. Vol. 1, Edición 1:30-43, Reino Unido.

PROYECTO DE APOYO A LA INNOVACIÓN TECNOLÓGICA (2004). Innovadores. Año 1, No.

1, Septiembre. Managua: Ministerio de Fomento Industria y Comercio.

ROBERTS, P. W. \& AMIT, R. (2003). “The Dynamics of Innovative Activity and Competitive Advantage: The Case of Australian Retail Banking 1981 to 1995”. En Organization Science. 14:107-122, Maryland. 